\title{
Projectile Two-dimensional Coordinate Measurement Method Based on Optical Fiber Coding Fire and its Coordinate Distribution Probability
}

\author{
Hanshan Li, Zhiyong Lei \\ School of Electronic Information Engineering, Xi’an Technological University, 710032, Xi'an ,P.R.China, \\ E-mail: lihanshan269@163.com
}

\begin{abstract}
To improve projectile coordinate measurement precision in fire measurement system, this paper introduces the optical fiber coding fire measurement method and principle, sets up their measurement model, and analyzes coordinate errors by using the differential method. To study the projectile coordinate position distribution, using the mathematical statistics hypothesis method to analyze their distributing law, firing dispersion and probability of projectile shooting the object center were put under study. The results show that exponential distribution testing is relatively reasonable to ensure projectile position distribution on the given significance level. Through experimentation and calculation, the optical fiber coding fire measurement method is scientific and feasible, which can gain accurate projectile coordinate position.
\end{abstract}

Keywords: Optical fiber coding fire, projectile, firing coordinate, mathematical statistics, distributing probability

\section{INTRODUCTION}

$\mathrm{W}$ ITH THE DEVELOPMENT of artillery technology, the assessment of indicators on artillery technology will be more strict. In shooting range, the dynamic parameters of a flying projectile are a very important index in the weapon testing field, such as velocity, coordinate, stance, firing dispersion, and so on [1-3]. These parameters will restrict manufacture and development of new weapons. Especially the index of firing dispersion on artillery shooting projectile is a very important parameter, which is estimate indicator of tactical and technical performance of weapon systems [5-6]. Now, artillery can shoot an object precisely, which is the ultimate development goal to modern weapons, so, many countries are very concerned about fire intensity in weapon systems. There are many influence factors in systems measuring firing dispersion, such as the composing artillery system condition, ammunition condition, object detection and precision calculation, launch site condition, weather conditions, and so on [7]. These restricting conditions are interrelated and influence each other, if we want to research the artillery shooting dispersion we have to study a new measurement projectile coordinate and distribution probability arithmetic. The projectile position has certain distribution, which is decided by firing dispersion. To study flying projectile coordinate parameters and firing dispersion, we have to study a new measurement method to gain the projectile position. This paper introduces the optical fiber coding fire measurement method, sets up their measurement model, and analyzes coordinate errors.

\section{PRoJeCtile COORDINATE MEASUREMENT PRINCIPLE BASED ON OPTICAL FIBER CODING FIRE}

Optical fiber coding fire measurement system is composed of optical fiber coding, array photoelectric detector, lens, and image and data processing system. We use two optical fiber coding system form intersection detection screens. When the projectile is flying into the detection screen, we calculate their coordinate position according to the measurement geometric model. The coordinate calculation principle of optical fiber coding fire measurement system is illustrated in Fig.1.

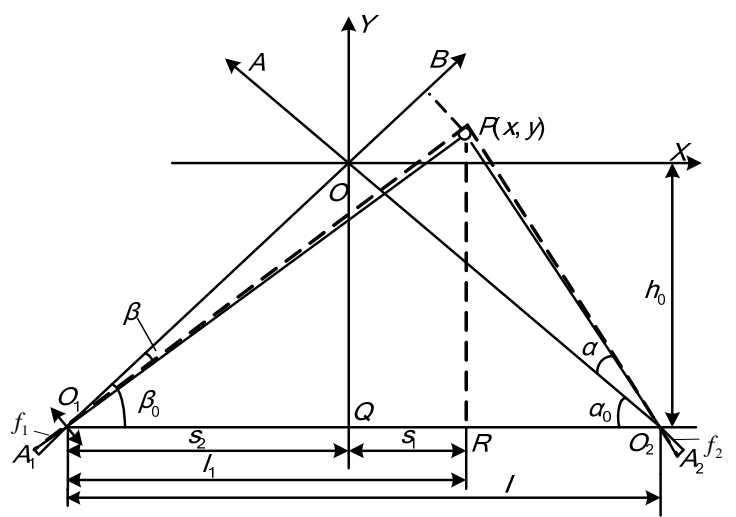

Fig.1 The coordinates calculation principle of optical fiber coding fire

In Fig.1, $O_{1}$ and $O_{2}$ are centers of lens of optical fiber coding fire, and they are positioned on both sides of the ballistic trajectory. $O_{1} A$ and $O_{2} B$ are their optical axes in the calculation model, $X O Y$ is their coordinate system, $O$ is shoot center, $\alpha_{0}$ is an angle between $O_{1} A$ and horizontal plane, $\beta_{0}$ is an angle between $O_{2} B$ and horizontal plane. Supposed, $P(x, y)$ is a coordinate point when a projectile is passing through detection screen, $\alpha$ is an angle between $O_{1} P$ and $O_{1} A, \beta$ is an angle between $O_{2} P$ and $O_{2} B$, $O_{2} Q=s_{2}, Q R=s_{1}, \quad A_{1}$ and $A_{2}$ are the width of projectile image in fiber coding, $Q O_{1}$ and $l_{1}$ can be obtained by formulas (1) and (2) according to the geometric relations in Fig.1. 


$$
\begin{gathered}
Q O_{1}=\frac{s_{1}+s_{2}}{1+\tan \left(\alpha_{0}+\alpha\right) / \tan \left(\beta_{0}-\beta\right)} \\
l_{1}=s_{1}+s_{2}
\end{gathered}
$$

According to Fig.1, assuming that $f_{1}=f_{2}=f$, projectile position coordination $P(x, y)$ can be calculated by formulas (3) and (4).

$$
x=\frac{l \times \tan \left[\beta_{0}-\arctan \left(\frac{A_{2}}{f}\right)\right]}{\tan \left[\beta_{0}-\arctan \left(\frac{A_{2}}{f}\right)\right]+\tan \left[\alpha_{0}-\arctan \left(\frac{A_{1}}{f}\right)\right]}-s_{2} . .
$$

$$
y=\frac{l \times \tan \left[\beta_{0}-\arctan \left(\frac{A_{2}}{f}\right)\right] \times \tan \left[\alpha_{0}-\arctan \left(\frac{A_{1}}{f}\right)\right]}{\tan \left[\beta_{0}-\arctan \left(\frac{A_{2}}{f}\right)\right]+\tan \left[\alpha_{0}-\arctan \left(\frac{A_{1}}{f}\right)\right]}-h_{0} \cdot
$$

Here, $h_{0}$ is the height of points $O$ to $O_{1} O_{2}$.

\section{THE ARITHMETIC PROJECTILE POSITION DISTRIBUTION PROBABILITY}

Based on the measurement data of the optical fiber coding fire measurement system, we use mathematical statistics hypothesis testing to analyze its position distribution probability.

We gained $n$ projectile coordinate data, such as $\left(x_{1}, y_{1}\right)$, $\left(x_{2}, y_{2}\right), \ldots,\left(x_{n}, y_{n}\right)$. To discuss the projectile position distribution law, fire dispersion and probability of the projectile shooting the object center under the conditional probability density function $\varphi(x \mid y)$, we use normal distribution hypothesis testing, exponential distribution hypothesis testing, and Rayleigh distribution hypothesis testing to analyze and ensure a reasonable hypothesis testing According to (5), we can calculate $y$ direction coordinate average value $\bar{y}$, and then, the conditional probability density of $\varphi(x \mid y)$ can be calculated under $n$ sample data, their statistical order is $x(i)(i=1, \ldots, n)$ [8-9]. Based on the statistical order and function $\varphi(x \mid y)$, we can calculate the projectile position distribution law.

$$
\bar{y}=\frac{1}{n} \sum y_{i}
$$

In shooting range, we gain two team data $A$ and $B, A$ order statistical values $x_{1 i}$ are $-1.35,-1.08,-1.02,-0.37$, $-0.22,-0.18,-0.15,0.23,0.52,0.68,0.87$, the average value is $\bar{y}_{1}$. $B$ order statistical values $x_{2 i}$ are $-1.67,-1.33$,
$-1.18,-1.05,0.08,0.15,0.28,0.53,1.06,1.23,1.42$, their average value is $\bar{y}_{2}$.

\section{A. Normal distribution testing}

The projectile position distribution law $\varphi(x \mid y)$ is perceived as normal distribution [10]. Its function can be expressed by equation (6).

$$
\varphi(x \mid y)=\frac{1}{\sqrt{2 \pi}} e^{-(x-\mu)^{2} / 2 \sigma^{2}}
$$

Here, $y$ is average value. The testing statistic of $W$ is

$$
W=\left[\sum_{i=1}^{n / 2} a_{i}[x(n-i+1)-x(i)]\right]^{2} /\left[\sum_{i=1}^{n}\left[x(i)-\bar{x}^{2}\right]\right.
$$

In (7), $a_{i}(1 \leq i \leq[n / 2])$ can be found in Table 1 . When sample number $n$ is equal to 11 , the value of $a$ is given in Table 2.

Table 1. The value of $a$

\begin{tabular}{|l|l|l|l|l|}
\hline$a_{1}$ & $a_{2}$ & $a_{3}$ & $a_{4}$ & $a_{5}$ \\
\hline 0.560 & 0.3315 & 0.2260 & 0.1429 & 0.0695 \\
\hline
\end{tabular}

Table 2. The value of $W_{a}(11)$

\begin{tabular}{|l|l|}
\hline$a$ & $W_{a}(11)$ \\
\hline $1 \%$ & 0.792 \\
$5 \%$ & 0.850 \\
$10 \%$ & 0.876 \\
\hline
\end{tabular}

If the normal distribution assumption is set up, the distribution of $W$ is only related to the sample's capacity $n$. Given that the significance level $a$ is known, assumption is refused when $W \leq W_{a}(n) \cdot W_{a}(n)$ is shown in Table 2. The value of $W$ can be calculated by (7), based on two team data $A$ and $B$, and the calculation principle, $W_{A}$ is $0.835, W_{B}$ is 0.821 . According to Table 2, assumption is refused when $a \geq 5 \%$, because $W_{A}$ and $W_{B}$ are less than 0.850 , and it meets the condition $W \leq W_{a}(n)$. So, the position distribution law of projectile cannot be seen as normal distribution.

\section{B. Rayleigh distribution testing}

The projectile position distribution law $\varphi(x \mid y)$ is presumed as Rayleigh distribution, it can be expressed by equation (8).

$$
\varphi(x \mid \bar{y})=\frac{b-x}{a^{2}} e^{-(b-x)^{2} / 2 a^{2}} \quad x<b
$$

When $x \geq b, \varphi(x \mid \bar{y})=0$.

Make the transformation $y=-(b-x)^{2}$. 
According to the statistical nature of maximum order statistic $x(n)$ and moment estimation of parameter $a$, unbiased estimation $b$ is calculated by equation (9) , [11].

$$
\hat{b}=[\sqrt{n} x(n)-\bar{x}] /(\sqrt{n}-1)
$$

New order statistics and observations can be obtained by making the transformation $y(i)=-(3.208-x)^{2}$.

The testing statistic is expressed as follows:

$$
x^{2}=-2 \sum_{k=2}^{n-1} \ln \left(S_{k} / S_{1}\right)
$$

Here, $S_{k}$ and $T_{i}$ can be gained by equations (11) and (12).

$$
\begin{gathered}
S_{k}=\sum_{i=k}^{n-1} T_{i}(k=1, \cdots, n-1) \\
T_{i}=i[x(i+1)-x(i)] \quad(i=1, \cdots, n-1)
\end{gathered}
$$

If the Rayleigh distribution assumption is set up, $x^{2}$ distribution's freedom is $2(n-1)$, given the significance level $a$ is known. When $x^{2}>x^{2}{ }_{a / 2}[2(n-1)]$ or $x^{2}<x_{1-(a / 2)}^{2}[2(n-1)]$, assumption is refused. $\chi^{2}(18)$ and $\chi^{2}(20)$ can be found in Table 3 .

Table 3. The value of $x^{2}(18)$ and $x^{2}(20)$

\begin{tabular}{|c|c|c|}
\hline$a$ & $\chi^{2}(18)$ & $\chi^{2}(20)$ \\
\hline 0.900 & 10.86 & 12.44 \\
\hline 0.800 & 12.86 & 14.58 \\
\hline 0.750 & 13.68 & 15.45 \\
\hline 0.600 & 15.89 & 17.81 \\
\hline 0.500 & 17.34 & 19.34 \\
\hline 0.400 & 18.87 & 20.95 \\
\hline 0.250 & 21.60 & 23.83 \\
\hline 0.200 & 22.76 & 25.04 \\
\hline 0.100 & 25.99 & 28.41 \\
\hline
\end{tabular}

Based on two team data $A$ and $B$, the value of $x^{2}$ can be calculated, $X_{A}{ }^{2}$ equals to 24.12, $x_{B}{ }^{2}$ equals to 25.92 . According to $\chi^{2}(n)$ distribution theory and Table 3, $n=11$, when $a>0.2$, assumption of Rayleigh distribution is refused. So the projectile position distribution law cannot be seen as Rayleigh distribution.

\section{Exponential distribution testing}

The position distribution law of center of seals deviating from optical axis $\varphi(x \mid y)$ is presumed as exponential distribution [12-13]. Its function can be expressed by equation (13).

$$
\varphi(x \mid y)=\left\{\begin{array}{cc}
\frac{1}{a} e^{-[(b-x) / a]} & x<\mathrm{b} \\
0 & x \geq \mathrm{b}
\end{array}\right.
$$

The testing statistics is expressed:

$$
x^{2}=-2 \sum_{k=2}^{n-1} \ln \left(S_{k} / S_{1}\right)
$$

In (14),

$$
\begin{aligned}
& S_{k}=\sum_{i=k}^{n-1} T_{i} \quad(\mathrm{k}=1, \ldots, \mathrm{n}-1) \\
& T_{\mathrm{i}}=i[x(i+1)-x(i)] \quad(\mathrm{i}=1, \ldots, \mathrm{n}-1)
\end{aligned}
$$

If the exponential distribution assumption is set up, the freedom of $x^{2}$ distribution is $2(n-2)$. Given the significance level $a$ is known, assumption is refused when $x^{2}>x_{a / 2}^{2}[2(n-2)]$ or $x^{2}<x_{1-(a / 2)}^{2}[2(n-2)]$. The value of $x^{2}$ can be calculated, $x_{\mathrm{A}}^{2}$ is $17.23, x_{\mathrm{B}}{ }^{2}$ is 16.36, According to $\chi^{2}(n)$ distribution theory and Table 3 , $n=11$, we can see that even if $a$ is more than 0.6 , the assumption can be accepted. So, the projectile burst position distribution law can be seen as exponential distribution.

According to the above analysis, we can use exponential distribution to calculate projectile position distribution probability.

\section{Projectile shooting object center probability analysis}

In the optical fiber coding fire measurement system, the position of the projectile's hitting object offset is the main parameter, it affects the projectile object damage efficiency. In shooting aim, the center of object is the artillery aim point, this point is named as shooting area. Suppose point $O$ is center of shooting coordinate in Fig.1, the side length of shooting area is $l_{x}$ in $x$ direction, the side width is $l_{y}$ in $y$ direction, so the shooting area can be decided by $l_{x} \times l_{y}$.

Supposed, $\Phi(y)$ is density function, it can be expressed by equation (15).

$$
\Phi(y)=2 \int_{0}^{y} e^{-t^{2} / 2} / \sqrt{2 \pi} d t
$$

If $P(x, y)$ is the aim point when shooting a gun, $\left(E_{-}, E_{-}\right)$

is initialization firing accuracy, the firing dispersion is ( $E_{x}, E_{y}$ ), and then the probability of first projectile hit can be calculated by equation (16), [14].

$$
\begin{aligned}
p= & \frac{1}{4}\left\{\Phi\left[\left(x+l_{x}\right) / E_{x}\right]-\Phi\left[\left(x-l_{x}\right) / E_{x}\right]\right\} \\
& \times\left\{\Phi\left[\left(y+l_{y}\right) / E_{y}\right]-\Phi\left[\left(y-l_{y}\right) / E_{y}\right]\right\}
\end{aligned}
$$


In (16), the relation of $\left(E_{-}, E_{y}\right), \quad\left(E_{x}, E_{y}\right)$ and $E_{X}, E_{Y}$ can be denoted by equation (17).

$$
\left\{\begin{array}{l}
E_{X}^{2}=E_{x}^{2}+E_{x}^{2} \\
E_{Y}^{2}=E_{y}^{2}+E_{y}^{2}
\end{array}\right.
$$

Based on the above theory, we can calculate the projectile shooting object center offset probability.

\section{PROJECTILE COORDINATE ERROR ANALYSIS}

If we ignore $S_{2}$ and $h_{0}$ influence according to (3) and (4), we can use the differential method to analyze their error. Their differential expression can be calculated by equation (18) and (19).

$$
\begin{aligned}
d x= & \frac{\partial x}{\partial l} d l+\frac{\partial x}{\partial \alpha_{0}} d \alpha_{0}+\frac{\partial x}{\partial \beta_{0}} d \beta_{0}+\frac{\partial x}{\partial A_{1}} d A_{1} \\
& +\frac{\partial x}{\partial f} d f+\frac{\partial x}{\partial A_{2}} d A_{2} \\
d y= & \frac{\partial y}{\partial l} d l+\frac{\partial y}{\partial \alpha_{0}} d \alpha_{0}+\frac{\partial y}{\partial \beta_{0}} d \beta_{0}+\frac{\partial y}{\partial A_{1}} d A_{1} \\
& +\frac{\partial y}{\partial f} d f+\frac{\partial y}{\partial A_{2}} d A_{2}
\end{aligned}
$$

If the shooting area is about $10 \mathrm{~m} \times 10 \mathrm{~m}$, $\mathrm{h}_{0}=2 \mathrm{~m}, \mathrm{~s}_{2}=16 \mathrm{~m}, \mathrm{~d} \alpha=d \beta=0.0002 \mathrm{rad}, \mathrm{dl}=0.5 \mathrm{~mm}$, the number of single optical fiber image systems is 4096, the size of every optical fiber is $0.01 \mathrm{~mm}$, the focus of lens is $50 \mathrm{~mm}$, df $=0.02 \mathrm{~mm}$. According to the differential expression, we can estimate their errors using Matlab, the distributing curve of $|d x|$ and $|d y|$ is shown in Fig.2 and Fig.3, the result shows the highest error of $|d x|$ and $|d y|$, which are about $12 \mathrm{~mm}$ and $25 \mathrm{~mm}$, respectively.

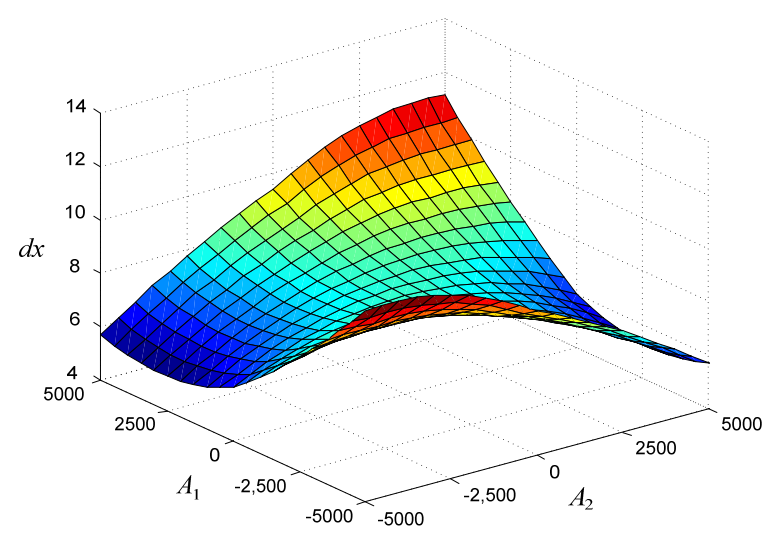

Fig.2. The distributing curve of $|d x|$

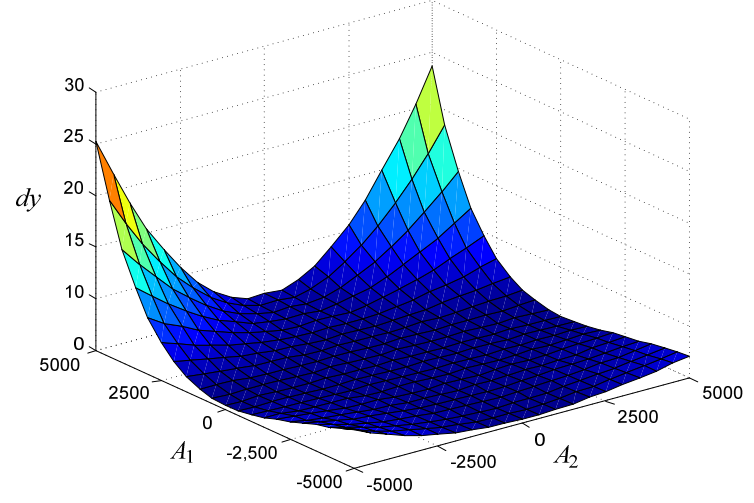

Fig.3. The distributing curve of $|d y|$

\section{EXPERIMENT AND ANALYSIS}

Based on the above principle and analysis, we use the four-screen measurement system and optical fiber coding fire measurement system to measure the same projectile coordinate. The four-screen measurement system uses four screens across to form the measurement area. Table 4 and Table 5 are the measurement data obtained by the fourscreen measurement system and optical fiber coding fire measurement system.

Table 4. The measurement coordinate data on optical fiber coding fire measurement system

\begin{tabular}{|l|l|l|l|l|l|l|}
\hline No. & $x$ & $y$ & $x_{p}$ & $y_{p}$ & $\Delta x$ & $\Delta y$ \\
\hline 1 & -1267 & -2108 & -1270 & -2115 & 3 & 7 \\
\hline 2 & -1342 & -2004 & -1345 & -2013 & 3 & 9 \\
\hline 3 & -2087 & -1785 & -2094 & -1792 & 7 & 7 \\
\hline 4 & -1762 & -1293 & -1770 & -1285 & 8 & -8 \\
\hline 5 & -1908 & -2507 & -1918 & -2515 & 10 & 8 \\
\hline 6 & -2004 & -1098 & -1997 & -1103 & -7 & 5 \\
\hline 7 & -1855 & -2036 & -1862 & -2045 & 7 & 9 \\
\hline
\end{tabular}

Table 5. The measurement coordinate data on four-screen measurement system

\begin{tabular}{|l|l|l|l|l|l|l|}
\hline No. & $x_{w}$ & $y_{w}$ & $x_{p}$ & $y_{p}$ & $\Delta x_{w}$ & $\Delta y_{w}$ \\
\hline 1 & -1289 & -2127 & -1270 & -2115 & -19 & -12 \\
\hline 2 & -1366 & -2030 & -1345 & -2013 & -21 & -17 \\
\hline 3 & -2085 & -1782 & -2094 & -1792 & 9 & 10 \\
\hline 4 & -1783 & -1297 & -1770 & -1285 & -13 & -12 \\
\hline 5 & -1933 & -2530 & -1918 & -2515 & -15 & -15 \\
\hline 6 & -2008 & -1126 & -1997 & -1103 & -11 & -23 \\
\hline 7 & -1876 & -2055 & -1862 & -2045 & -14 & -10 \\
\hline
\end{tabular}

Here, the coordinates of the four-screen measurement system method are $x_{w}$ and $y_{w}, \Delta x_{w}$ and $\Delta y_{w}$ are their errors. The coordinates of the optical fiber coding fire measurement system are $x$ and $y, \Delta x$ and $\Delta y$ are their errors. $x_{p}$ and $y_{p}$ are the coordinates where a flying projectile drills through a board, which we can read $x_{p}$ and $y_{p}$ directly on the board by tapeline, measurement data units are millimeter in both cases, in optical fiber coding fire measurement system, $\mathrm{l}=24 \mathrm{~m}, \mathrm{~h}_{0}=5 \mathrm{~m}, \alpha=\beta=45^{\circ}$. 
From Table 4 and Table 5, the average error between the optical fiber coding fire measurement system and the board coordinate on projectile two-dimensional coordinate system are less than $10 \mathrm{~mm}$, the average error between the fourscreen measurement system and the board coordinate on projectile two-dimensional coordinate system is about 23 mm. By comparison of the optical fiber coding fire measurement method and four-screen across system, the result shows the optical fiber coding fire measurement system improves the measurement precision.

In addition, according to the probability theory of object hit and Table 4 and Table 5, if the middle coordinate is $O(0,0)$, here we design $l_{x}$ and $l_{y}$ as $2 \mathrm{~m}$, the first firing accuracies $E_{\bar{x}}$ and $E_{\bar{y}}$ are $1.207 \mathrm{~m}$ and $2.016 \mathrm{~m}$, their firing dispersions $E_{x}$ and $E_{y}$ are $0.734 \mathrm{~m}$ and $1.314 \mathrm{~m}$. Thus, we can calculate other parameters, and the team data probability of the first projectile hitting object is $24.2 \%$.

Based on the exponential distribution assumption, the freedom of $x^{2}$ distribution is $2(n-2)$, here $n=7, x_{1}^{2}$ is 16.25. According to $\chi^{2}(n)$ distribution theory, we can see that even if $\alpha$ is more than 0.78 , the assumption can be accepted. The result is in accordance with exponential distribution.

\section{CONCLUSIONS}

This paper introduces the optical fiber coding fire measurement system and sets up their measure geometric mathematical model. According to the calculation expression, the differential method was used to analyze its measurement error; the method of mathematical statistics was used to analyze the projectile position distribution probability. Discussed was the normal distribution hypothesis testing, exponential distribution hypothesis testing, Rayleigh distribution hypothesis testing, the projectile position distribution law view as $\varphi(x \mid y)$ function, based on measurement data and hypothesis testing, the projectile position distribution law was perceived as exponential distribution under some given significance level $\alpha$, the probability of projectile shooting the object center was analyzed, and the tool of Matlab was applied to describe the error variety curve. The results show that the optical fiber coding fire measurement system meets the test demands, this measurement method has been applied successfully in the range, which has gained good achievement.

\section{ACKNOWLEDGMENT}

This work has been supported by Project of the National Natural Science Foundation of China (61107079).

\section{REFERENCES}

[1] Dong, T., Ni, J., Ma, S. (2007). A Method of computing the pill flying time based on sky screens. Journal of Detection \& Control, 29 (3), 29-33.
[2] Li, H., Lei, Z. (2009). Principle and analysis of high altitude projectile position measurement using multiscreen target method. Chinese Journal of Scientific Instrument, 30 (3), 621-625.

[3] Wik, T., Gustafsson, H. (1993). A new method to calculate trajectories of base bleed projectiles. In Ballistics 2003 : 14th International Symposium in Canada, 16-18 August 1993, 737-744.

[4] Li, H., Gao, H. (2007). Research on improve the photoelectric detecting performance of optic-electric detecting targets. Trajectory Journal, 19 (1), 33-36.

[5] Jiang, M., Li, H. (2006). Analysis of the performances of optic-electric detecting target optical system. Xi'an Institute of Technology Academic Journal, 26 (2), 127130.

[6] Ni, J., Yang, L., Tian, H. (2008). Measurement principle for two kinds of six-light-screen array composed by a large area light screen. Opto-Electronic Engineering, 35 (1), 7-12.

[7] Li, H., Lei, Z., Wang, Z. (2007). A new type of firing accuracy measurement system for cross sky screen. Electronics Optics \& Control, 14 (5), 153-154.

[8] Liu, Q., Shi, H. (2004). Precision analysis on infrared intensity photoelectric vertical targets. Optical Technology, 30 (6), 758-760.

[9] Zeng, G.Y., Ma, Y. (2004). Studies on measuring system of erecting target. Journal of Projectile, Rockets, Missiles and Guidance, 25 (1), 345-346.

[10] Li, H., Lei, Z. (2009). The study and design of antilight interference in PDT. In 2nd International Conference on Intelligent Computing Technology and Automation, 26-28 April 2009. Changsha, China, 711714.

[11] Lu, S.T., Yu, A.T., Chou, C. (1993). Electro-optics target for position and speed measurement. In IEEE Proceedings-A, 140 (4), 252-256.

[12] Hilt, B., Fessler, P., Prevot, G. (1999). High-speed data acquisition system for spectroscopic imaging of ionizing particles. IEEE Transactions on Nuclear Science, 46 (5), 1285-1288.

[13] Li, H., Lei, Z., Wang, Z. (2012). Research on objection information extraction arithmetic in photo-electric detection target base on wavelet analysis method. Przeglad Elektrotechniczny (Electrical Review), 88 (9), 157-161.

[14] Ding, L., Zhang, H., Zhou, X. (2005). Study on muzzle velocity precision measurement of high-peed projectile. In The 6th International Symposium on Test and Measurement, 20-21 January 2005. Beijing, China, 811-814.

Received June 10, 2012. Accepted January 25, 2013. 